\title{
Stable high-order finite-difference methods based on non-uniform grid point distributions
}

\author{
Miguel Hermanns and Juan Antonio Hernández \\ E.T.S.I. Aeronauticos, Universidad Politecnica de Madrid, Plaza Cardenal Cisneros 3, Madrid 28040, Spain
}

\begin{abstract}
SUMMARY
It is well known that high-order finite-difference methods may become unstable due to the presence of boundaries and the imposition of boundary conditions. For uniform grids, Gustafsson, Kreiss, and Sundström theory and the summation-by-parts method provide sufficient conditions for stability. For non-uniform grids, clustering of nodes close to the boundaries improves the stability of the resulting finite-difference operator. Several heuristic explanations exist for the goodness of the clustering, and attempts have been made to link it to the Runge phenomenon present in polynomial interpolations of high degree.

By following the philosophy behind the Chebyshev polynomials, a non-uniform grid for piecewise polynomial interpolations of degree $q \leqslant N$ is introduced in this paper, where $N+1$ is the total number of grid nodes. It is shown that when $q=N$, this polynomial interpolation coincides with the Chebyshev interpolation, and the resulting finite-difference schemes are equivalent to Chebyshev collocation methods. Finally, test cases are run showing how stability and correct transient behaviours are achieved for any degree $q<N$ through the use of the proposed non-uniform grids. Discussions are complemented by spectra and pseudospectra of the finite-difference operators.
\end{abstract}

KEY WORDS: high-order scheme; finite difference; piecewise polynomials; stability; Runge phenomenon; pseudospectra

\section{INTRODUCTION}

The rise of computers and their use to solve physical problems numerically have constantly increased the relevance of numerical methods. This has led over the past decades to a continuous search for easier and better methods with greater accuracy and stability. High-order numerical methods present much less error than second-order methods for the same number of nodes or degrees of freedom. The benefits in terms of required resources and computational time to achieve a 
certain level of accuracy are clear Additionally, problems characterized by small length scales cannot be addressed nowadays without the use of high-order numerical methods. Computational acoustics and aeroacoustics, prediction of transition and direct numerical simulation of turbulence are typical examples of such problems

Depending on the regularity of the solutions, different numerical methods have been developed. While finite-volume, finite-element, and spectral Galerkin methods are obtained from the integral formulation of the problem, finite-difference and spectral collocation methods are derived from the differential form. Finite-difference and spectral collocation methods seek to enforce the system of partial differential equations of the problem on a discrete set of collocation nodes. To approximate the derivatives in the equations, an interpolant is built using the nodal values. The nature of the interpolant and of the chosen collocation nodes gives rise to different numerical methods, like Fourier and Chebyshev collocation methods and finite-difference methods

To analyse the stability of difference operators, different approaches have to be followed depending on the nature of the problem. For normal problems, the behaviour of the system is fully characterized by the spectrum of the operator. Therefore, the absence of eigenvalues with positive real parts ensures stability and bounded errors at all times [8]. In contrast, for non-normal problems, the eigenvalues are only relevant for the asymptotic behaviour of the system, allowing arbitrarily large transient growth at finite times. To overcome this limitation, the pseudospectra of the operator have to be studied, leading to necessary and sufficient conditions for stability at all times [8].

The instabilities of finite-difference methods have been related to the way they treat boundary conditions. Various approaches have been proposed to cure the problem. When uniform grids are used, GKS (Gustafsson, Kreiss, and Sundström) theory provides a sufficient test for stability and a recipe for obtaining stable boundary treatments that do not cause radiation of spurious waves into the computational domain Another approach, based on the energy method and the boundness of the numerical energy, leads to modified stable finite-difference schemes which are known as summation-by-parts methods (SBP) When non-uniform grids are used, clustering of nodes close to the boundaries cures the instability problems of high-order numerical methods This observation has prompted the use of clustered nodes at the boundaries by several authors

The approximation of smooth functions by polynomial interpolations on equispaced nodes can give rise to vigorous oscillations of the interpolant near the boundaries. This undesired effect is known as Runge phenomenon A well-known remedy to this problem is to use the Chebyshev roots as interpolation nodes, which minimize these undesired oscillations and lead to well-behaved polynomial interpolations. Some progress has been made to explain the goodness of clustering in finite-difference methods through the Runge phenomenon However, the question of which node distributions should be used is still not answered.

The purpose of this paper is to develop stable high-order finite-difference methods based on nonuniform grids, which are derived by following the philosophy behind the Chebyshev polynomials of minimizing the vigorous oscillations associated to the Runge phenomenon.

The paper is organized as follows. Section 2 reformulates the finite-difference methods in terms of piecewise polynomial interpolations on non-uniform grids. Then, Section 3 discusses the different factors contributing to the interpolation errors, showing that the use of Chebyshev roots as grid points leads to minimum errors. Section 4 presents the algorithm for obtaining the non-uniform grids that minimize the error of piecewise polynomial interpolations, followed 
by discussions of the resulting interpolation errors and node distributions. Thereafter, Section 5 analyses the spatial discretization errors resulting from the discretization of differential operators. The performance of the use of the proposed grids is shown in Section 6 by solving several normal and non-normal problems. The wave equation with reflecting boundary conditions is studied in Section 6.1, while Section 6.2 compares the proposed finite-difference methods with the high-order numerical methods proposed by Zhong and Tatineni using the one-dimensional convection equation. Then, Section 6.3 deals with the non-normal convection-diffusion operator and its transient solutions. Section 6.4 compares the proposed finite-difference methods with the SBP method proposed by Mattsson and Nordström the Chebyshev collocation method, and the mapped Chebyshev collocation methods proposed by Kosloff and Tal-Ezer and analyses their computational cost for the solution of a convection-diffusion problem. Finally, Section 6.5 extends the proposed grids to multiple dimensions by solving the wave equation in a two-dimensional square box.

\section{FINITE DIFFERENCES VIA PIECEWISE POLYNOMIAL INTERPOLATIONS}

Consider the following initial boundary value problem for $u(x, t)$ given by the evolution equation:

$$
\frac{\partial u}{\partial t}=\mathscr{L}\left(u, \frac{\partial u}{\partial x}, \frac{\partial^{2} u}{\partial x^{2}}\right)
$$

and the boundary and initial conditions

$$
\begin{gathered}
\mathscr{B}_{1}(u, \partial u / \partial x)=0, \quad x=-1 \\
\mathscr{B}_{2}(u, \partial u / \partial x)=0, \quad x=+1 \\
u(x, 0)=f(x) \quad \forall x \in[-1,+1]
\end{gathered}
$$

Equation (1) allows to evolve $u(x, t)$, constrained by the boundary conditions (2), from the initial condition (3) to a desired time $t>0$.

An approximate solution to the formulated differential problem is obtained by discretizing the spatial domain $[-1,+1]$ in $N+1$ nodes $\left\{x_{i}, i=0, \ldots, N\right\}$ and considering the corresponding nodal values $u_{i}(t)$ as unknowns of the semidiscrete problem, whose temporal evolution is governed by the following system of ordinary differential equations:

$$
\frac{\mathrm{d} u_{i}}{\mathrm{~d} t}=L_{i}\left(u_{0}, u_{1}, \ldots, u_{N}\right), \quad i=1, \ldots, N-1
$$

and the following constraints and initial conditions:

$$
\begin{gathered}
B_{1}\left(u_{0}, u_{1}, \ldots, u_{N}\right)=0, \quad i=0 \\
B_{2}\left(u_{0}, u_{1}, \ldots, u_{N}\right)=0, \quad i=N \\
u_{i}(0)=f\left(x_{i}\right), \quad i=0, \ldots, N
\end{gathered}
$$

To obtain the difference operator (4) and boundary conditions (5) that approximate their differential counterparts (1) and (2), a piecewise polynomial interpolant for the sufficiently smooth 


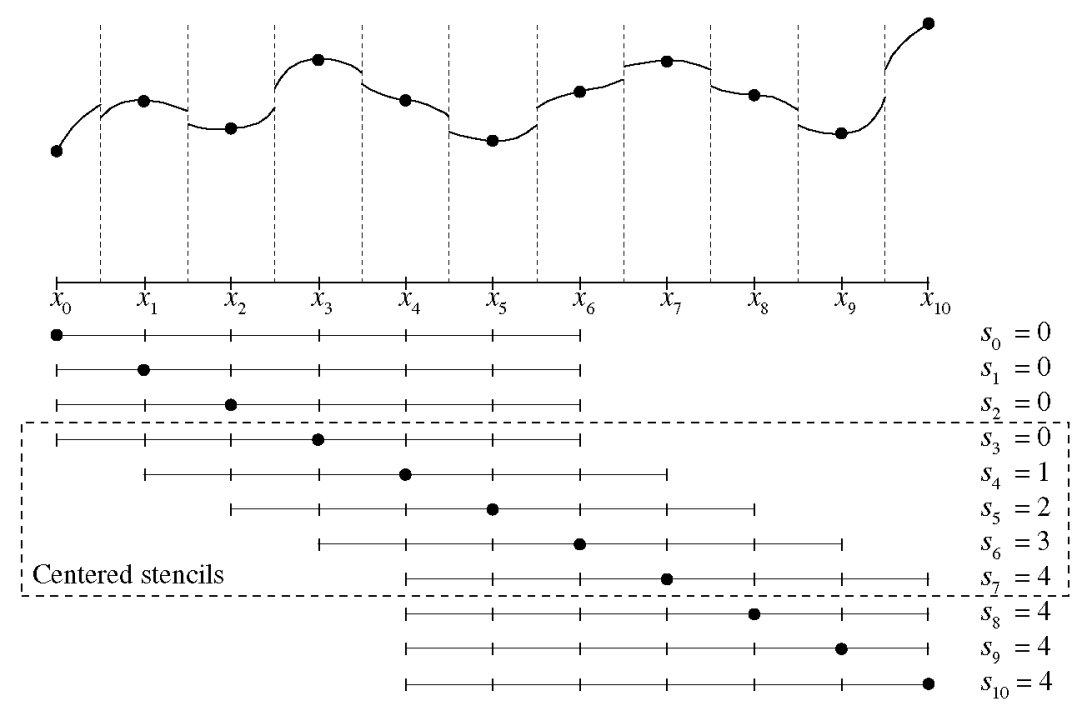

Figure 1. Stencils of the individual interpolants $I_{i}(x)$ for a piecewise polynomial interpolation of degree $q=6$ on 11 nodes $(N=10)$. The dashed box separates the centred stencils from those affected by the presence of the boundaries.

function $u(x, t)$ is constructed that matches the discrete values $u_{i}(t)$ at $x_{i}$. This interpolant is used to approximate the derivatives of $u(x, t)$ at the nodes $x_{i}$, leading finally to the semidiscretized problem (4)-(6).

Each of the individual interpolants $I_{i}(x)$ that form the piecewise approximation is only valid in a subdomain $\Omega_{i}$ that includes the node $x_{i}$ and is defined as

$$
\Omega_{i}:\left[x_{i-1 / 2}, x_{i+1 / 2}\right), \quad i=0, \ldots, N
$$

where the subdomain limits $x_{i-1 / 2}$ and $x_{i+1 / 2}$ are not relevant for the moment and, therefore, their definition is postponed. These interpolating polynomials of degree $q \leqslant N$ are built by forcing them to satisfy a set of $q+1$ distinct pairs of values $\left(x_{j}, u_{j}(t)\right)$. The resulting expressions for $I_{i}(x)$ can easily be obtained through the Lagrange interpolation formulae

$$
I_{i}(x)=\sum_{j=s_{i}}^{s_{i}+q} \ell_{i j}(x) u_{j}, \quad \ell_{i j}(x)=\prod_{\substack{m=0 \\ s_{i}+m \neq j}}^{q} \frac{x-x_{s_{i}+m}}{x_{j}-x_{s_{i}+m}}
$$

For centred finite-difference formulas, the polynomial degree $q$ has to be chosen even, and

$$
\left\{s_{i}\right\}=\{\underbrace{0, \ldots, 0}_{q / 2 \text { times }}, \underbrace{0,1, \ldots, N-q}_{\text {centred FD }}, \underbrace{N-q, \ldots, N-q}_{q / 2 \text { times }}\}
$$

The resulting finite-difference expressions for the derivatives of $u(x, t)$ will be centred for nodes $x_{i}$ sufficiently far away from the boundaries of the interval $\left[x_{0}, x_{N}\right]$, whereas close to the boundaries these expressions will be biased towards the centre of the interval, as can be seen in Figure 1, which shows the stencils of the interpolants for the case $q=6$ and $N=10$. 
If the polynomial degree $q$ is chosen odd instead, then

$$
\left\{s_{i}\right\}=\{\underbrace{0, \ldots, 0}_{(q-1) / 2 \text { times }}, \underbrace{0,1, \ldots, N-q}_{\text {uncentred FD }}, \underbrace{N-q, \ldots, N-q}_{(q+1) / 2 \text { times }}\}
$$

and the resulting finite-difference expressions will be slightly uncentred to the right.

\section{INTERPOLATION ERRORS ASSOCIATED WITH A GIVEN GRID}

The pointwise error between $u(x, t)$ and the piecewise polynomial interpolant is defined as

$$
\varepsilon_{i}(x)=u(x, t)-I_{i}(x) \quad \forall x \in \Omega_{i}
$$

Again, a simple expression for $\varepsilon_{i}(x)$ can be obtained through the Lagrange interpolation formulae for the case of sufficiently smooth functions [28]:

$$
\varepsilon_{i}(x)=\pi_{i}(x) \frac{u^{(q+1)}(\xi)}{(q+1) !}
$$

where $\xi=\xi(x)$ is some point in the interval $\left[x_{s_{i}}, x_{s_{i}+q}\right]$ and $\pi_{i}(x)$ is the following polynomial of degree $q+1$ :

$$
\pi_{i}(x)=\prod_{m=0}^{q}\left(x-x_{s_{i}+m}\right)
$$

Two different factors contribute to the pointwise error $\varepsilon_{i}(x)$ in (12): (i) the $(q+1)$ st derivative of the interpolated function $u(x, t)$ and (ii) the polynomial factor $\pi_{i}(x)$. In order to reduce the interpolation error, nothing can be done to improve the regularity of the function to interpolate, whereas the magnitude of $\pi_{i}(x)$ can be minimized by properly selecting the interpolation nodes $x_{i}$.

To enlighten the discussion, the particular case $q=N$ is considered next, for which only one polynomial can be constructed out of the $N+1$ interpolation nodes and, therefore, all the interpolants $I_{i}(x)$ collapse into one, as well as the polynomial factors $\pi_{i}(x)=\pi(x)$.

It is well known that the use of equally spaced nodes $x_{i}$ leads to large oscillations of $\pi(x)$ near the boundaries of the interval $\left[x_{0}, x_{N}\right]$, and therefore to large interpolation errors. This undesired effect, known as Runge phenomenon, can be observed in Figure 2(a), where the absolute value of $\pi(x)$ is represented on the interval $[-1,+1]$ for the case $N=10$. The extrema of $\pi(x)$, which are located between adjacent nodes, reach significantly larger values close to the boundaries than far from them.

The question regarding which nodes minimize the oscillations of the polynomial factor was addressed by Chebyshev who demonstrated that the use of the Chebyshev roots as interpolation nodes leads to interpolations with the lowest achievable pointwise error. Chebyshev showed that a necessary and sufficient condition for this property is that all the extrema of $|\pi(x)|$ reach the same value. If $y_{1}, \ldots, y_{N}$ represent the roots of the Chebyshev polynomial $T_{N}(x)$, then an interpolant of degree $N-1$ based on these nodes presents minimum oscillations in $\pi(x)$. This can be seen in Figure 2(b).

The main drawback of the Chebyshev roots is the absence of the locations $x= \pm 1$, which is an inconvenience for the enforcement of boundary conditions at the limits of the interval. Therefore, 

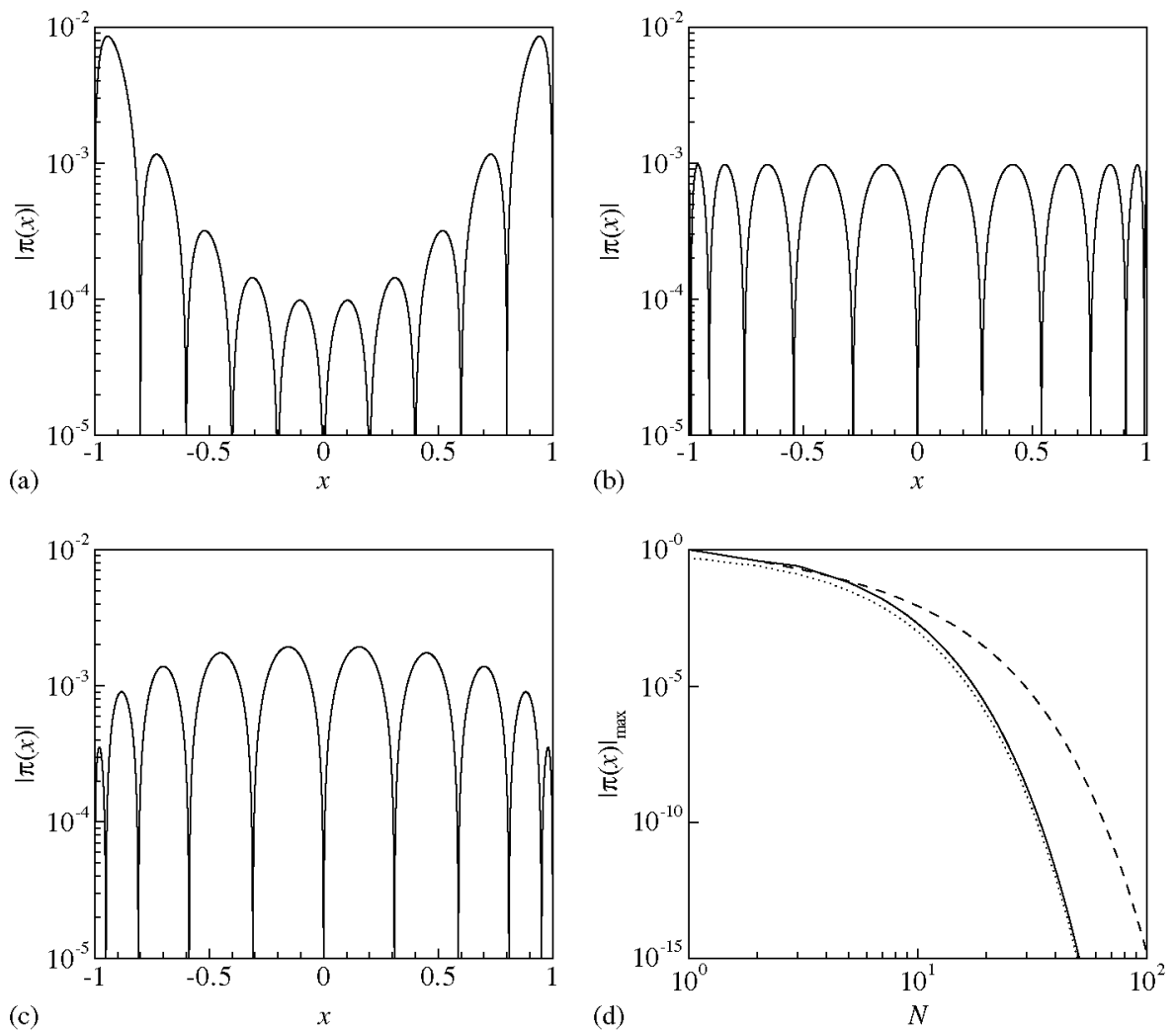

Figure 2. Absolute value of the polynomial factor $\pi(x)$ for $q=N=10$ : (a) equispaced nodes; (b) Chebyshev roots; and (c) Chebyshev extrema. (d) Rate of decay of $|\pi(x)|_{\max }$ with the number of nodes $N$ : Chebyshev roots (dotted), Chebyshev extrema (solid), and equispaced nodes (dashed).

a more convenient set of nodes has to be found. If $x_{0}, \ldots, x_{N}$ denote the Chebyshev extrema of $T_{N}(x)$, which include $x_{0}=-1$ and $x_{N}=+1$, then an interpolant of degree $N$ based on these nodes behaves almost as good as the one based on the Chebyshev roots Although the resulting polynomial factor $\pi(x)$ does not oscillate uniformly, as can be seen in Figure 2(c), both sets of nodes present similar convergence properties. This is shown in Figure 2(d), where the rate of decay of the maximum value of $|\pi(x)|$ is represented. The committed error using equispaced nodes is several orders of magnitude larger than when using Chebyshev roots or Chebyshev extrema.

The next section will proceed similarly and apply the same philosophy to the generic case $q<N$.

\section{NON-UNIFORM GRIDS FOR PIECEWISE POLYNOMIAL INTERPOLATIONS WITH UNIFORM ERROR}

When $q<N$, neither the Chebyshev roots nor the Chebyshev extrema minimize the error of the piecewise polynomial interpolation. To find the equivalent grid for $q<N$, two piecewise polynomial interpolations are considered: one of degree $q-1$ based on the unknown nodes $y_{1}, \ldots, y_{N}$ 
(equivalent to the Chebyshev roots when $q=N$ ), and a second one of degree $q$ based on the extrema $x_{0}, \ldots, x_{N}$ (equivalent to the Chebyshev extrema when $q=N$ ) of the first one, which include $x_{0}=-1$ and $x_{N}=+1$. By imposing equal maximum amplitudes for all the polynomial factors $\pi_{i}(x)$, the equivalent grid $x_{0}, \ldots, x_{N}$ is obtained.

The algorithm can be summarized as follows:

1. Consider $N$ unknown nodes $y_{1}, \ldots, y_{N}$ in the interval $[-1,+1]$ and build out of them a piecewise polynomial interpolation of degree $q-1$.

2. Look for the extremum of each polynomial factor $\pi_{i}(x)$ in its subdomain $\left[y_{i}, y_{i+1}\right)$

$$
\pi_{i}^{\prime}(x)=0, \quad i=1, \ldots, N-1
$$

and label the extrema as $x_{1}, \ldots, x_{N-1}$. Complete the set with the locations $x_{0}=-1$ and $x_{N}=+1$.

3. Impose equal amplitudes at the extrema of the polynomial factors:

$$
\left|\pi_{1}\left(x_{0}\right)\right|=\left|\pi_{1}\left(x_{1}\right)\right|=\left|\pi_{2}\left(x_{2}\right)\right|=\cdots=\left|\pi_{N-1}\left(x_{N-1}\right)\right|=\left|\pi_{N-1}\left(x_{N}\right)\right|
$$

Since the locations of the extrema $x_{1}, \ldots, x_{N-1}$ depend on $y_{1}, \ldots, y_{N}$, the above conditions represent a non-linear system of $N$ equations for the $N$ unknowns $y_{1}, \ldots, y_{N}$.

4. Known the values of $y_{1}, \ldots, y_{N}$, build the piecewise polynomial interpolation of degree $q$ using $x_{0}, \ldots, x_{N}$ as collocation nodes. The subdomains for the different interpolants will be

$$
\Omega_{0}=\left[-1, y_{1}\right), \quad \Omega_{i}=\left[y_{i}, y_{i+1}\right), \quad \Omega_{N}=\left[y_{N},+1\right]
$$

which finally gives the definition for the subdomain limits (7) introduced before.

The non-linear system of equations (15) has to be solved using a Newton-Raphson method. Since the radius of convergence of this method is relatively small, the solution is obtained through a continuation method in $q$. The resulting algorithm takes, on a standard personal computer (Pentium IV or similar), of the order of seconds to obtain the solution for typical values of $N=\mathscr{O}(100)$ and $q=\mathscr{O}(10)$. Taking into account that the grid point distribution only needs to be precomputed once and for all, its cost is clearly negligible when compared with the total cost of a typical numerical simulation.

In order to see the aspect of the resulting grids, consider a piecewise polynomial interpolation of degree $q=6$ on $N=10$ nodes. Figure 3(a) shows the absolute value of the polynomial factors $\pi_{i}(x)$ in their respective subdomains $\Omega_{i}$. The general shape resembles the one in Figure 2(c), which corresponds to $q=N=10$ using Chebyshev extrema. The clearly visible discontinuities in Figure 3(a) are located at the subdomain limits $y_{i}$ and are caused by a change of subdomain $\Omega_{i}$ and interpolating polynomial, in accordance with the stencils given in Figure 1. The amplitude of these discontinuities can be obtained by comparing two polynomial factors $\pi_{i}(x)$, of two consecutive subdomains, evaluated at the common subdomain limit $y_{i}$ :

$$
\begin{aligned}
\left|\pi_{i}\left(y_{i}\right)\right|-\left|\pi_{i-1}\left(y_{i}\right)\right| & =\left[\left|y_{i}-x_{s_{i}+q}\right|-\left|y_{i}-x_{s_{i-1}}\right|\right] \prod_{m=0}^{q-1}\left|y_{i}-x_{s_{i}+m}\right| \\
& =\left[1-\frac{\left|y_{i}-x_{s_{i-1}}\right|}{\left|y_{i}-x_{s_{i}+q}\right|}\right]\left|\pi_{i}\left(y_{i}\right)\right|
\end{aligned}
$$



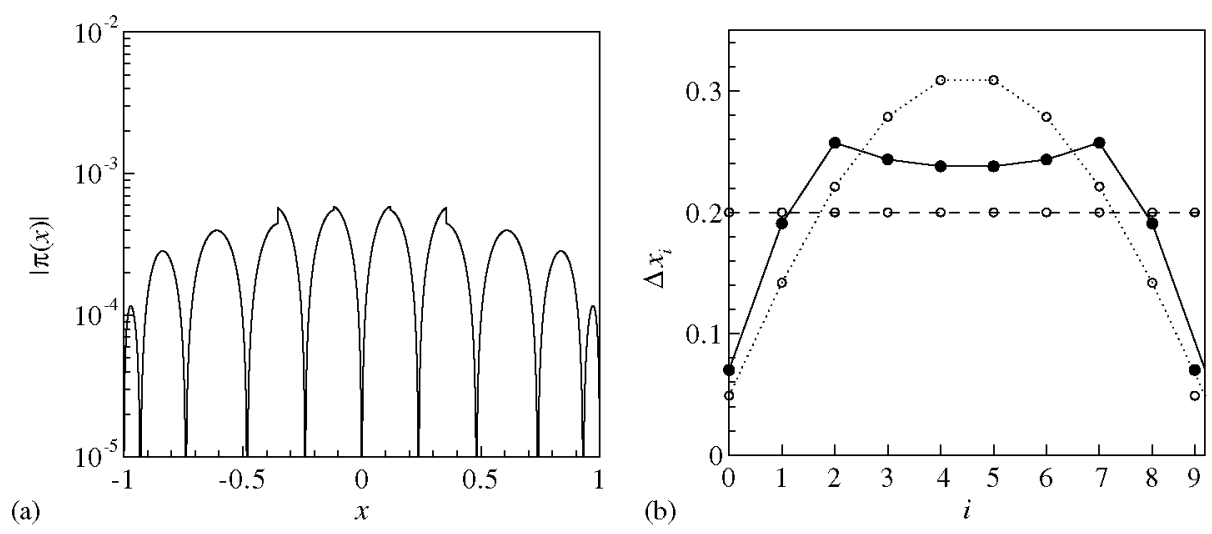

Figure 3. (a) Values of $\left|\pi_{i}(x)\right|$ in their respective subdomains $\Omega_{i}$ for the proposed non-uniform grid with $q=6$ and $N=10$. (b) Grid spacing $\Delta x_{i}=x_{i+1}-x_{i}$ of the proposed non-uniform grid (solid), the Chebyshev extrema (dotted), and the equispaced grid (dashed).

where the fact that the two polynomial factors only differ by one root has been taken into account. Two different factors contribute to the amplitude of these discontinuities: (i) the relative distance to $y_{i}$ of the farthest nodes involved in the interpolations and (ii) the amplitude of the polynomial factor. For smoothly varying grid point distributions, the first factor is small, and the jumps at the subdomain limits are smaller than the interpolation error of $\mathcal{O}\left(\left|\pi_{i}\left(y_{i}\right)\right|\right)$. It is important to note that these discontinuities are not located at the nodes $x_{i}$ on which the interpolant is used to approximate the derivatives and, therefore, are not seen by the finite-difference method. Figure 3(b) shows the corresponding grid spacing $\Delta x_{i}=x_{i+1}-x_{i}$. The proposed non-uniform grids present their maximum grid spacing off the centre of the domain. This unexpected result has no further consequences on the interpolation error, as the amplitudes of the polynomial factor in Figure 3(a) show.

Two different limiting cases are very enlightening: (i) $q=N$ and (ii) $q \ll N$. In the first case, the proposed algorithm matches the steps followed in the previous section and, therefore, returns the extrema of $T_{N}(x)$ as collocation nodes $x_{0}, \ldots, x_{N}$ and the roots of $T_{N}(x)$ as subdomain limits $y_{1}, \ldots, y_{N}$. Since there is a unique interpolating polynomial of degree $N$, the expressions for the Lagrange interpolation and the discrete Chebyshev series coincide, and all the properties of the Chebyshev collocation methods are enforceable, specially their spectral accuracy In the second case, when $q \ll N$, only a few points $\mathscr{O}(q)$ close to the boundaries need to be displaced in order to control the oscillations of the pointwise error. Far from them, the influence of the boundaries is negligible and the resulting node distribution is equispaced. This can be seen in Figure 4, where 4(a) shows the absolute value of the polynomial factors $\pi_{i}(x)$ in their respective subdomains $\Omega_{i}$ and $4(\mathrm{~b})$ the grid spacing $\Delta x_{i}=x_{i+1}-x_{i}$ of the proposed non-uniform grid, both for a piecewise polynomial interpolation of degree $q=6$ on $N=30$ nodes.

Finally, when $q<N$, most of the nodes are affected by the presence of the boundaries and the resulting node distributions are in between the two limiting cases. This can be seen in Figure 5(a), where the grid spacing of the proposed non-uniform grids for different values of $q$ and $N=50$ is shown. As the degree of the interpolation increases, the node distribution approaches the Chebyshev 

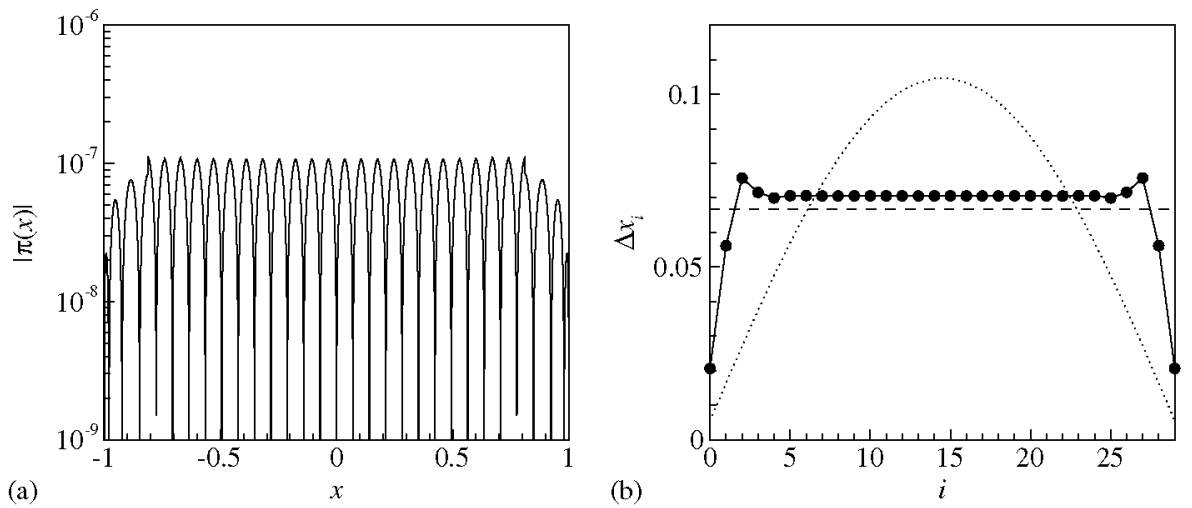

Figure 4. (a) Values of $\left|\pi_{i}(x)\right|$ in their respective subdomains $\Omega_{i}$ for the proposed non-uniform grid with $q=6$ and $N=30$. (b) Grid spacing $\Delta x_{i}=x_{i+1}-x_{i}$ of the proposed non-uniform grid (solid), the Chebyshev extrema (dotted), and the equispaced grid (dashed).
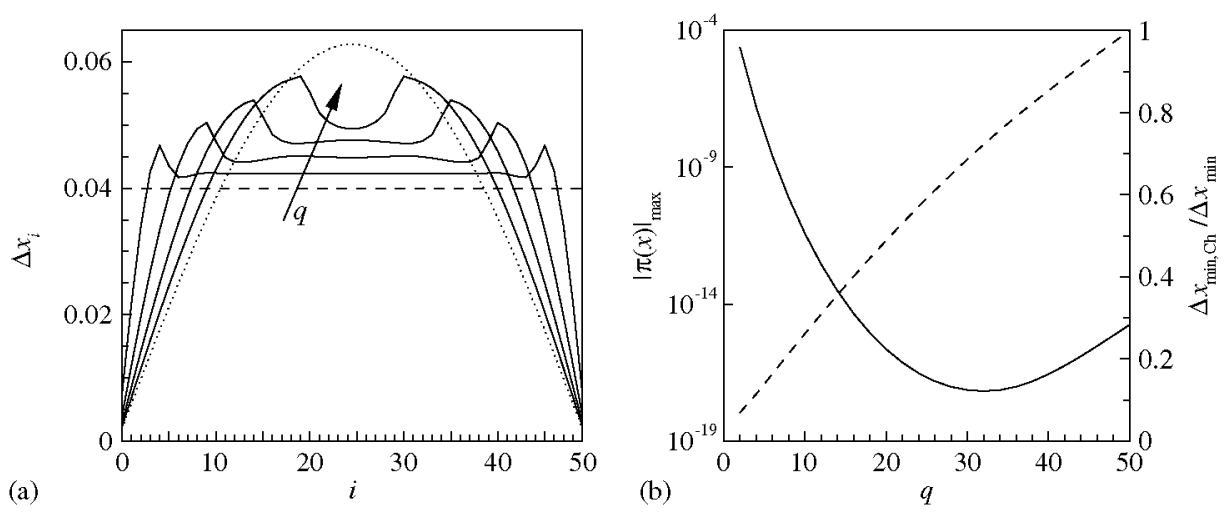

Figure 5. (a) Grid spacing of the proposed non-uniform grid (solid) for $N=50$ and $q=10,20,30,40$, the Chebyshev extrema (dotted), and the equispaced grid (dashed). (b) Variation of $|\pi(x)|_{\max }$ (solid) and $\Delta x_{\min , \mathrm{Ch}} / \Delta x_{\min }$ (dashed) with $q$ for the proposed non-uniform grid with $N=50$.

extrema distribution, while for small values of $q$ it is more close to the equispaced one. Figure 5(b) shows how the maximum value of the polynomial factors $\left|\pi_{i}(x)\right|$ varies with $q$ for $N=50$. The presence of a minimum in $|\pi(x)|_{\max }$ of three orders of magnitude lower than for the Chebyshev interpolation shows that the best interpolation is achieved for $q<N$. Figure 5(b) also shows that the minimum grid spacing $\Delta x_{\min }$ is always greater than the minimum grid spacing $\Delta x_{\min , \mathrm{Ch}}$ of the Chebyshev extrema: $\Delta x_{\min }=\mathcal{O}\left(\Delta x_{\min , \mathrm{Ch}} N / q\right)=\mathscr{O}\left((q N)^{-1}\right)$.

The clustering of grid nodes caused by the use of the proposed non-uniform grids is independent of the problem to be solved, and has only to do with the interpolation, just as in Chebyshev collocation methods. 


\section{SPATIAL DISCRETIZATION ERRORS}

Until now, only the interpolation errors of piecewise polynomial interpolations were of concern. But small interpolation errors do not necessarily mean small spatial discretization errors, as these may grow unbounded or present anomalous transient behaviours due to spatial discretizations [20].

The spatial discretization errors are defined as the differences between the exact solution $u(x, t)$ at the collocation nodes and the solution $u_{i}(t)$ of the semidiscrete problem (4)-(6):

$$
E_{i}(t)=u\left(x_{i}, t\right)-u_{i}(t), \quad i=1, \ldots, N-1
$$

A system of equations for their temporal evolution can be obtained by subtracting (4) from (1) at the collocation nodes. For linear differential operators in (1),

$$
\frac{\mathrm{d} \mathbf{E}}{\mathrm{d} t}=\mathbf{A E}+\mathbf{R}
$$

where $\mathbf{A}$ is the $N-1 \times N-1$ matrix which is the difference operator with homogeneous boundary conditions, and $\mathbf{R}(t)$ is the $N-1$ column vector with the truncation errors, which for linear operators are given by

$$
R_{i}=\left.\mathscr{L}(\varepsilon)\right|_{x_{i}}, \quad i=1, \ldots, N-1
$$

being $\varepsilon(x)$ the interpolation error defined in (11). If $\mathbf{A}$ is constant, then (19) has the following analytical solution:

$$
\mathbf{E}(t)=\mathrm{e}^{\mathbf{A} t} \mathbf{E}(0)+\int_{0}^{t} \mathrm{e}^{\mathbf{A}(t-\tau)} \mathbf{R}(\tau) \mathbf{d} \tau
$$

Assuming that the initial condition is exact, the spatial discretization error at $t=T$ can be bounded by

$$
\|\mathbf{E}\| \leqslant \sup _{t \in[0, T]}\left\|\mathrm{e}^{\mathbf{A} t}\right\| \sup _{t \in[0, T]}\|\mathbf{R}\| T
$$

For vectors, $\|\cdot\|$ denotes the weighted vector norm that approximates the continuous $L^{2}$-norm on functions $[29,30]$, and for matrices it denotes the corresponding subordinate matrix norm. From a practical point of view, it is desirable that (22) is at most $\mathcal{O}(\|\mathbf{R}\| T)$ for a time of integration $T$, so that the spatial discretization error behaves with $N$ in the same way as the truncation error does. Therefore, an upper bound for $\left\|\mathrm{e}^{\mathbf{A} t}\right\|$ is necessary in order to know the accuracy of the scheme. In the most general case, the following upper bound in terms of the spectral abscissa $\alpha(\mathbf{A})$ can be obtained [8]:

$$
\left\|\mathrm{e}^{\mathbf{A} t}\right\| \leqslant \kappa \mathrm{e}^{\alpha t}, \quad \alpha(\mathbf{A})=\sup _{z \in \Lambda(\mathbf{A})} \operatorname{Re}(z)
$$

where $\Lambda(\mathbf{A})$ represents the spectrum of $\mathbf{A}$ and $\kappa(\mathbf{V})=\|\mathbf{V}\|\left\|\mathbf{V}^{-1}\right\|$ is the condition number of the matrix of eigenvectors $\mathbf{V}$ of $\mathbf{A}$.

When the spatial discretization of a given problem leads to a normal matrix $\left(\mathbf{A A}^{\mathrm{T}}=\mathbf{A}^{\mathrm{T}} \mathbf{A}\right)$, then $\kappa=1$ and the fundamental matrix is bounded by unity if $\alpha \leqslant 0$, which happens when all the eigenvalues of $\mathbf{A}$ have a negative real part. Otherwise, if $\alpha>0$, the norm of the fundamental matrix is not bounded as $t \rightarrow \infty$ and, consequently, the norm of the error grows without bound. 
When the spatial discretization of a given problem leads to a non-normal matrix $\left(\mathbf{A} \mathbf{A}^{\mathrm{T}} \neq \mathbf{A}^{\mathrm{T}} \mathbf{A}\right)$, the condition number $\kappa$ can be very large. In that case, for finite times $t=T$, the spatial discretization error may grow larger than the truncation error, even when $\alpha \leqslant 0$ (which now only ensures that the error goes to zero as $t \rightarrow \infty$ ). Therefore, for non-normal matrices it is not sufficient to analyse their eigenvalues to know the accuracy of the numerical method. To bound $\left\|\mathrm{e}^{\mathbf{A} t}\right\|$ and to assure small spatial discretization errors for finite times, the pseudospectra of the matrix $\mathbf{A}$ can be used. For each $\varepsilon \geqslant 0$, a subset of the complex plane, the $\varepsilon$-pseudospectrum of $\mathbf{A}$, is defined as follows [8]:

$$
\Lambda_{\varepsilon}(\mathbf{A})=\{z \in \mathbb{C}: z \in \Lambda(\mathbf{A}+\Delta \mathbf{A}) \text { for some } \Delta \mathbf{A} \text { with }\|\Delta \mathbf{A}\| \leqslant \varepsilon\}
$$

The $\varepsilon$-pseudospectrum of a matrix measures the sensitivity of its eigenvalues to perturbations of the matrix of amplitude $\|\Delta \mathbf{A}\| \leqslant \varepsilon$ [8]. If the matrix $\mathbf{A}$ is normal, the $\varepsilon$-pseudospectrum is exactly the set of points in the complex plane located at a distance $\leqslant \varepsilon$ from the eigenvalues of $\mathbf{A}$, but if the matrix is non-normal, it covers a much larger portion of the complex plane.

By making use of the Cauchy integral, the following upper bound can be obtained $[8,29,30]$ :

$$
\left\|\mathrm{e}^{\mathbf{A} t}\right\| \leqslant \frac{1}{2 \pi \varepsilon} \int_{\partial \Lambda_{\varepsilon}(\mathbf{A})} \mathrm{e}^{t \operatorname{Re}(z)}|\mathrm{d} z| \leqslant \frac{L_{\varepsilon}}{2 \pi \varepsilon} \mathrm{e}^{\alpha_{\varepsilon} t}
$$

where $\partial \Lambda_{\varepsilon}(\mathbf{A})$ is the boundary of $\Lambda_{\varepsilon}(\mathbf{A}), L_{\varepsilon}$ the arc length of $\partial \Lambda_{\varepsilon}(\mathbf{A})$, and $\alpha_{\varepsilon}(\mathbf{A})$ the s-pseudospectral abscissa of $\mathbf{A}$ :

$$
\alpha_{\varepsilon}(\mathbf{A})=\sup _{z \in \Lambda_{\varepsilon}(\mathbf{A})} \operatorname{Re}(z)
$$

which represents the rightmost point of the $\varepsilon$-pseudospectrum. If $\alpha_{\varepsilon}>0$, which means that the $\varepsilon$-pseudospectrum protrudes into the right half-plane, a lower bound for $\left\|\mathrm{e}^{\mathbf{A} t}\right\|$ can also be derived by means of the Laplace transform $[8,31]$ :

$$
\frac{\alpha_{\varepsilon}}{\varepsilon} \leqslant \sup _{t \geqslant 0}\left\|\mathrm{e}^{\mathbf{A} t}\right\|
$$

This lower bound combined with the upper bound (25) allows to know the magnitude of the norm of the fundamental matrix and with it the spatial discretization error of the numerical method:

$$
\frac{\alpha_{\varepsilon}}{\varepsilon} \leqslant \sup _{t \in[0, T]}\left\|\mathrm{e}^{\mathbf{A} t}\right\| \leqslant \frac{L_{\varepsilon}}{2 \pi \varepsilon} \mathrm{e}^{\alpha_{\varepsilon} t_{\max }}
$$

where $t_{\max }$ is the time, of order unity, at which the maximum value of $\left\|\mathrm{e}^{\mathbf{A} t}\right\|$ is achieved. Different values of $\varepsilon>0$ lead to different lower and upper bounds, and the largest and lowest of all of them, respectively, give precise bounds of the norm of the fundamental matrix.

In summary, when the spatial discretization of a given problem leads to a normal matrix $\mathbf{A}$, then the eigenvalue analysis of that matrix is enough to ensure stability and to bound the spatial discretization errors at all times $t$. But when the resulting matrix is non-normal, the eigenvalue analysis only ensures asymptotic stability and small errors for $t \rightarrow \infty$, and the pseudospectra analysis is required to obtain bounds for the spatial discretization errors at finite times $t=T$. 


\section{NUMERICAL EXAMPLES}

To show the goodness of the proposed non-uniform grids and their associated finite-difference schemes, three representative problems are solved: the wave equation and the convection equation as examples of normal problems and the convection-diffusion equation as an example of non-normal problem. Comparisons with other high-order numerical methods are made and the computational cost of the proposed finite-difference methods is analysed. Finally, the extension to multiple dimensions is demonstrated by solving multiple reflexions of a pressure wave inside a twodimensional rectangular box.

Time integration has been accomplished by a classical fourth-order Runge-Kutta method. Since only the spatial discretization errors are of interest, time steps have been chosen small enough to ensure negligible time integration errors.

\subsection{One-dimensional wave equation}

Consider the one-dimensional wave equation

$$
\frac{\partial^{2} p}{\partial t^{2}}=\frac{\partial^{2} p}{\partial x^{2}}
$$

with the following boundary and initial conditions,

$$
\begin{gathered}
\frac{\partial p}{\partial x}(0, t)=0, \quad \frac{\partial p}{\partial x}(1, t)=0 \\
p(x, 0)=\mathrm{e}^{-500(x-0.5)^{2}}, \quad \frac{\partial p}{\partial t}(x, 0)=0 \quad \forall x \in[0,1]
\end{gathered}
$$

Introducing the auxiliary variable $v=\partial p / \partial t$, the differential operator in (1) for the variables $(p, v)$ will be

$$
\mathscr{L}(p, v)=\left(v, \frac{\partial^{2} p}{\partial x^{2}}\right)^{\mathrm{T}}
$$

The interest in this test case, of hyperbolic character, resides in the existence of a temporal periodic solution which allows the exact determination of spatial discretization errors. In this case, the matrix A resulting from the spatial discretization of the problem is normal, which means that the eigenvalues of $\mathbf{A}$ determine its behaviour and that of the spatial discretization error

It is well known that when high-order explicit finite-difference methods are applied to hyperbolic problems on uniform grids, the discretized boundary conditions may lead to unstable difference operators This can be observed in Figure 6(a), where stable numerical solutions are obtained for $q \leqslant 6$ while unstable behaviours arise for $q \geqslant 8$. The instabilities come from the existence of eigenvalues with positive real parts, as shown in Figure $6(\mathrm{~b})$, where the spectrum of $\mathbf{A}$ for $q=8$ is shown.

The cure for these numerically induced instabilities can be found in the proposed non-uniform grids. Figure 7(a) compares different solutions at $t=10$ obtained with different degrees $q$, showing that stable behaviours are achieved for all values of $q$. Finally, Figure 7(b) shows the rate of decay of the maximum spatial discretization error until round-off with the number of nodes for different values of $q$. 

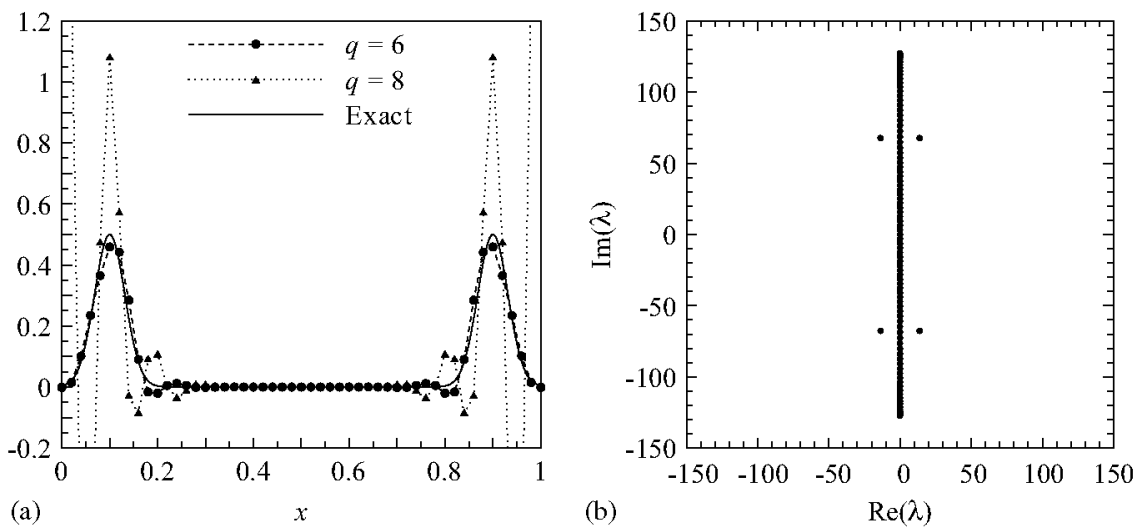

Figure 6. (a) Numerical solutions for the wave equation at $t=0.6$ obtained with $N=50$ and $\Delta t=0.0001$ on an equispaced grid. (b) Eigenvalues of the difference operator for $q=8$.
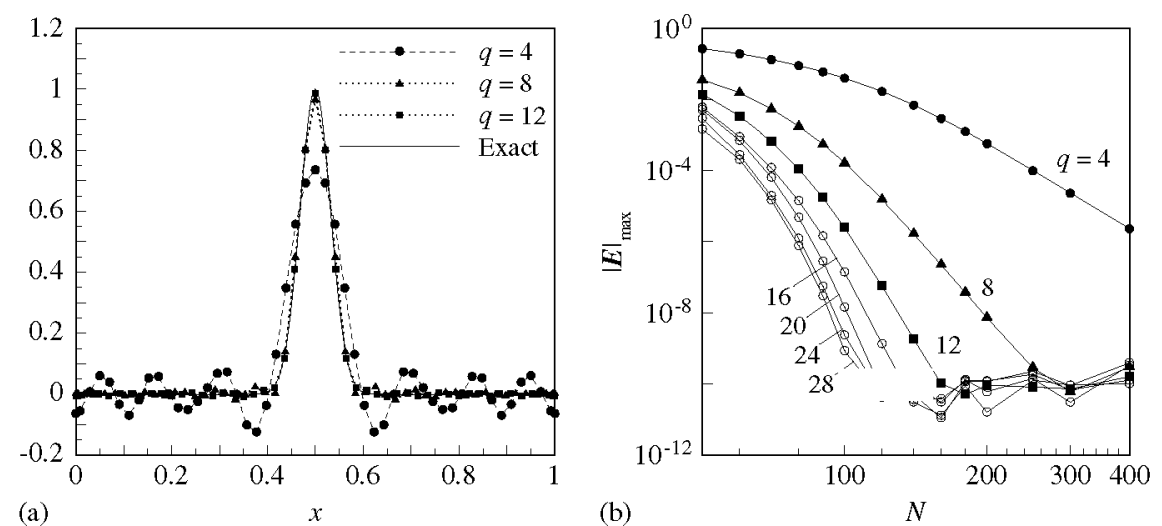

Figure 7. (a) Numerical solutions for the wave equation at $t=10$ obtained with $N=50$ and $\Delta t=0.0001$ using the proposed non-uniform grids. (b) Rate of decay of the maximum discretization error $|\mathbf{E}|_{\max }$ with $N$ for $q=4,8, \ldots, 28$.

\subsection{One-dimensional convection equation}

Consider the one-dimensional convection equation

$$
\frac{\partial u}{\partial t}=-\frac{\partial u}{\partial x}
$$

with the following boundary condition at the left end of the domain $[-1,1]$ :

$$
u(-1, t)=\sin (\pi t)
$$

This test case, proposed by Zhong and Tatineni [18], admits the following time periodic solution:

$$
u(x, t)=\sin (\pi(t-x-1))
$$



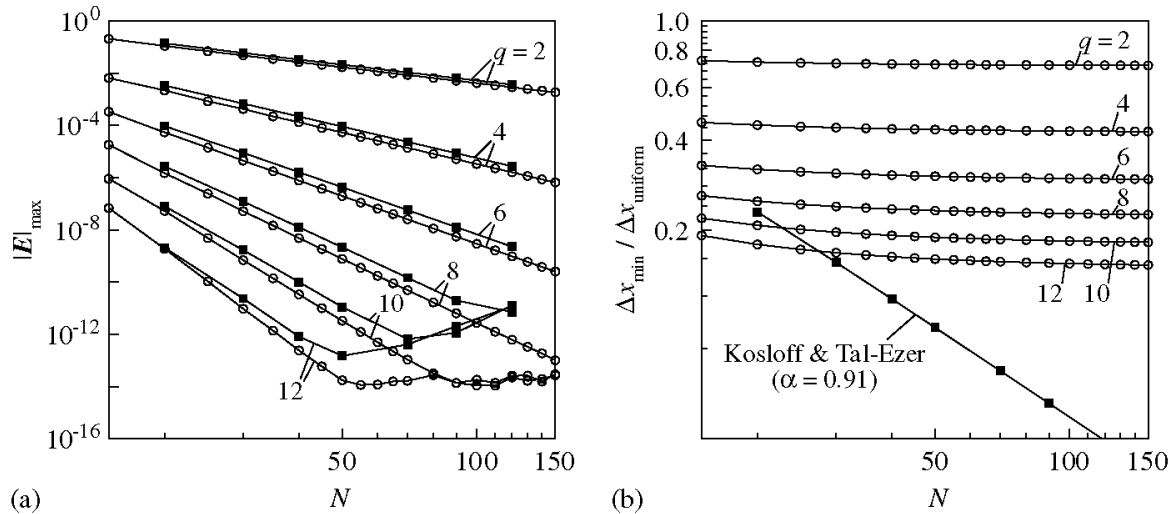

Figure 8. (a) Rate of decay of the maximum discretization error $|\mathbf{E}|_{\max }$ with $N$ for $q=2,4, \ldots, 12$ for the proposed non-uniform grids (hollow circles) and the Kosloff and Tal-Ezer grid with $\alpha=0.91$ used in [18] (filled squares). (b) Minimum grid spacing $\Delta x_{\min } / \Delta x_{\text {uniform }}$ as a function of $N$ and $q$ for the proposed non-uniform grids (hollow circles) and the grids used in (filled squares).

which allows the exact determination of the committed numerical errors. non-uniform grid point distribution, first introduced by Kosloff and Tal-Ezer alleviate the stability problems of high-order finite-difference methods:

$$
x_{i}=\frac{\sin ^{-1}(-\alpha \cos (\pi i / N))}{\sin ^{-1} \alpha}, \quad i=0, \ldots, N
$$

This distribution depends on the free parameter $\alpha$, which in the case of the computations performed in [18] is chosen to be $\alpha=0.91$. Figure 8 (a) shows the maximum spatial discretization error $|\mathbf{E}|_{\max }$ which results from the use of the different non-uniform grid point distributions. As in the previous case of the one-dimensional wave equation, the use of the proposed non-uniform grids leads to errors which decay until round-off with the number of nodes and for different values of $q$. Compared to Zhong and Tatineni, both grid point distributions lead to finite-difference methods which perform similarly in terms of spatial discretization errors, as Figure 8(a) indicates. Figure 8(b) shows the minimum grid spacings of the non-uniform grids employed in the computation of the results shown in Figure 8(a). Although is seems that the proposed non-uniform grids excel in this point, this is only true for the value of $\alpha$ used in $\quad$ By trial and error, the precise value of $\alpha$ required to make the numerical method stable can be obtained as a function of $N$ and $q \quad$ If these values of $\alpha$ are used, then the minimum grid spacings turn out to be quite similar. In view of these results, it can be inferred that the proposed non-uniform grid point distributions automatically incorporate the necessary amount of grid clustering at the boundaries to ensure the stability of the finite-difference approximation.

\subsection{One-dimensional convection-diffusion equation}

Consider the one-dimensional convection-diffusion equation

$$
\frac{\partial u}{\partial t}=\frac{\partial u}{\partial x}+\frac{\partial^{2} u}{\partial x^{2}}
$$



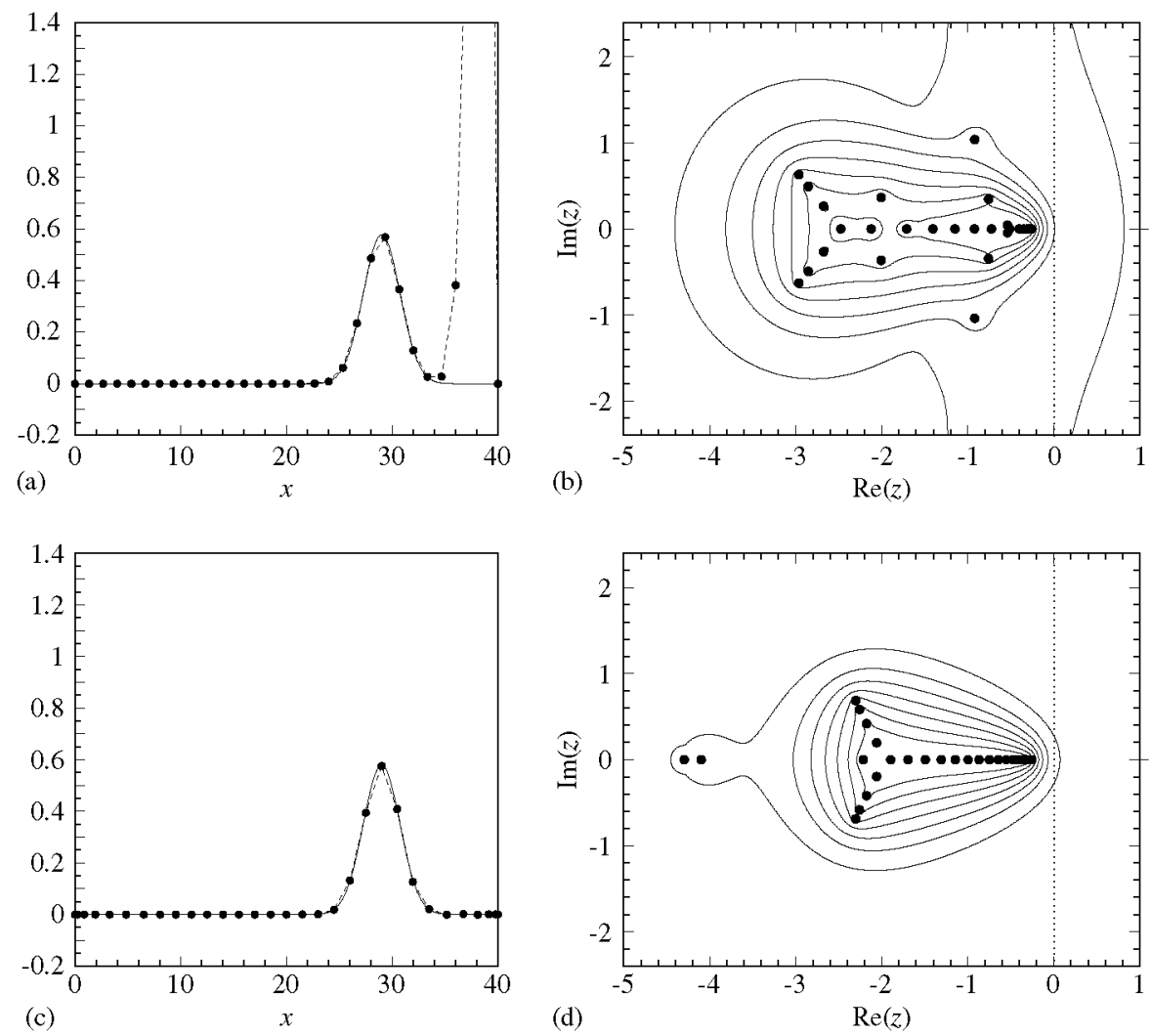

Figure 9. Transient behaviour of numerical solutions of the convection-diffusion equation with $d=40$ at $t=1$, obtained with $N=30, q=12$, and $\Delta t=0.0001$ on (a) an equispaced grid and (c) the proposed non-uniform grid. Eigenvalues (solid circles) and pseudospectra (solid lines) of the corresponding difference operator obtained on (b) an equispaced grid and (d) the proposed non-uniform grid. Contours at $\varepsilon=10^{-1}, 10^{-2}, \ldots, 10^{-7}$.

with the following boundary and initial conditions,

$$
\begin{gathered}
u(0, t)=0, \quad u(d, t)=0 \\
u(x, 0)=\mathrm{e}^{-(x-3 d / 4)^{2} / 2} \quad \forall x \in[0, d]
\end{gathered}
$$

The aim of this test case is to show the impact of different spatial discretizations on the prediction of transient behaviours of non-normal problems. The values $N=30$ and $d=40$ are chosen to allow direct comparisons with previous work done by Reddy and Trefethen

Figure 9(a) shows the numerical solution at $t=1$ obtained using a piecewise polynomial interpolation of degree $q=12$ on an equispaced grid. Although the numerical method is asymptotically stable, as the eigenvalues in Figure 9(b) indicate, the transient solution presents an anomalous peak that reaches a value of order 10 . The explanation of it can be found in the pseudospectra of the difference operator also shown in Figure 9(b), which significantly protrudes 

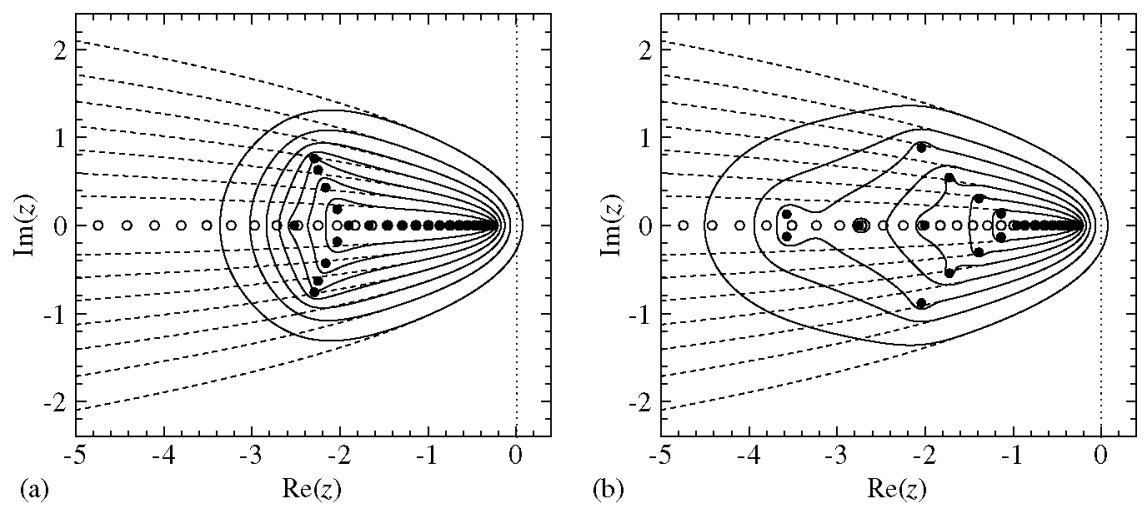

Figure 10. Eigenvalues (solid circles) and pseudospectra (solid lines) of the difference operator obtained from the convection-diffusion operator with $d=40$ using the proposed non-uniform grids with $N=30$ and (a) $q=14$ and (b) $q=30$ (Chebyshev). Contours at $\varepsilon=10^{-1}, 10^{-2}, \ldots, 10^{-7}$. Eigenvalues (empty circles) and pseudospectra (dashed lines) of the corresponding differential operator are also shown.

for $\varepsilon=10^{-1}$ into the right half-plane. The consequence of it are large lower and upper bounds for $\left\|\mathrm{e}^{\mathbf{A} t}\right\|$ as given by (28) allowing for spatial discretization errors larger than the truncation errors.

Instead, if the proposed non-uniform grids are used, the transient solution behaves correctly and the pseudospectra does not protrude anomaly into the right half-plane, as seen in Figure 9(c) and (d), leading to smaller lower and upper bounds for $\left\|\mathrm{e}^{\mathbf{A} t}\right\|$ and, consequently, to spatial discretization errors of the same order as the truncation errors.

In view of the results, it seems reasonable to expect, that a good spatial discretization not only approximates correctly the eigenvalues of the differential operator, but also its pseudospectra Reddy and Trefethen pointed out that good agreements in the vicinity of the imaginary axis correspond to smooth solutions that are well resolved by the discretization. Such good agreements can be observed in Figure 10, where the eigenvalues and pseudospectra of difference operators, obtained for different values of $q$, are shown together with the ones of the differential operator.

Remarkable is the fact that the finite-difference method using the proposed non-uniform grid and $q=14$ performs similar as the Chebyshev collocation method in terms of approximating the eigenvalues and pseudospectra of the differential operator.

\subsection{Computational cost}

The number of operations, or computational cost, of a numerical simulation is the product of the number of operations $N_{\text {ops }}$ related to the spatial discretization times the number of time steps required to reach a final simulation time.

The cost involved in the spatial discretization is mainly due to the required derivatives of the interpolant $I(x)$ at all the grid nodes. Once the coefficients $\ell_{i j}^{\prime}\left(x_{i}\right)$ in (8) have been precomputed, this cost is of order $q N$ for the proposed finite-difference methods For Chebyshev collocation methods, this cost is of order $5 N \log _{2} N$ due to the fast-Fourier transformations used for the computation of the derivatives 

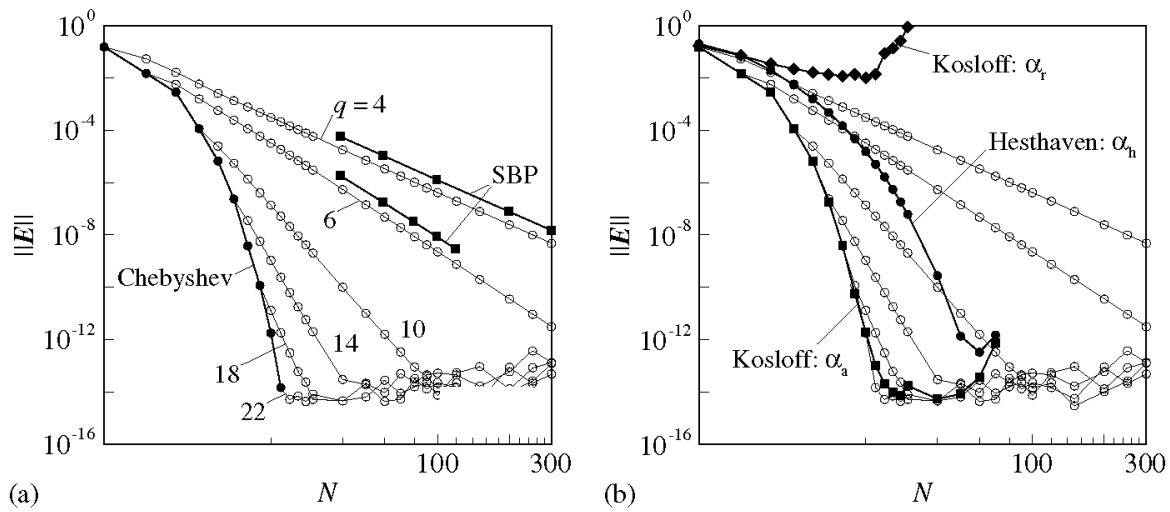

Figure 11. Comparison of spatial discretization errors $\|\mathbf{E}\|$ versus $N$ for the solution of the convection-diffusion problem proposed in using (a) Chebyshev collocation methods, summation-by-parts methods (SBP) the proposed finite-difference methods of degree $q$, and (b) the mapped Chebyshev collocation methods from with different criteria for the mapping parameter $\alpha$

Problem parameters are $\varepsilon=10^{-1}$ and $w=5 \sqrt{3}$.

Regarding the cost of the temporal discretization, time step is limited by error considerations and by stability constraints. Stability is ensured once the spectrum $\Lambda(\mathbf{A})$ of the spatial discretization, scaled by the time step $\Delta t$, is kept inside the stability region of the time integration method [6]. Hence, a useful measurement of the time step restriction to ensure stability is the spectral radius $\rho$ (A) [30]:

$$
\rho(\mathbf{A})=\sup _{\lambda_{i} \in \Lambda(\mathbf{A})}\left|\lambda_{i}\right|
$$

Once the stability constraint is fulfilled, the order of the temporal scheme is chosen such that temporal and spatial errors are of same order of magnitude.

The following convection-diffusion problem proposed in has been simulated to compare spatial errors and computational cost of the proposed finite-difference methods with those of the SBP methods of [16], the Chebyshev collocation methods, and the mapped Chebyshev collocation methods proposed by Kosloff and Tal-Ezer

$$
\begin{gathered}
\frac{\partial u}{\partial t}+\frac{\partial u}{\partial x}=\varepsilon \frac{\partial^{2} u}{\partial x^{2}} \\
-u(0, t)+\frac{\partial u}{\partial x}(0, t)=g_{0}(t), \quad \frac{\partial u}{\partial x}(1, t)=g_{1}(t) \\
u(x, 0)=f(x) \quad \forall x \in[0,1]
\end{gathered}
$$

where the functions $g_{0}(t), g_{1}(t)$, and $f(x)$ are chosen such that

$$
u(x, t)=\sin [w(x-c t)] \mathrm{e}^{-b x}, \quad c=\sqrt{1+4 w^{2} \varepsilon^{2}}, \quad b=\frac{c-1}{2 \varepsilon}
$$

is a time periodic solution. Figure 11 compares the spatial discretization errors $\|\mathbf{E}\|$ resulting from the use of the different numerical methods and different degrees $q$. The values of the parameters 

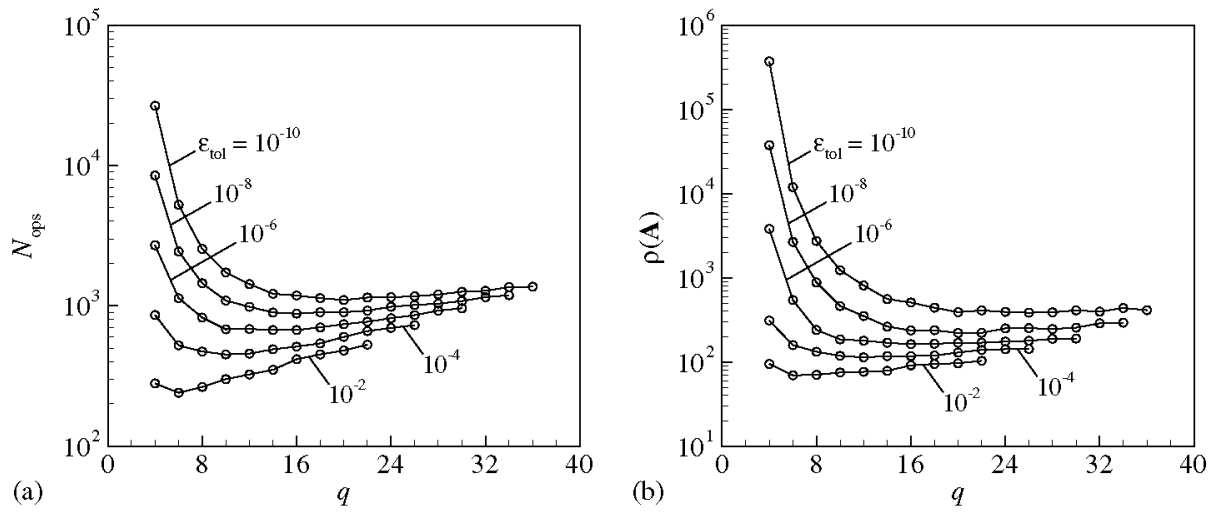

Figure 12. (a) Number of operations $N_{\mathrm{ops}}=\mathscr{O}(q N)$ and (b) spectral radius $\rho$ (A) of the spatial discretization of the convection-diffusion problem proposed as a function of $q$ for different error tolerances $\varepsilon_{\mathrm{tol}}$. Problem parameters are $\varepsilon=10^{-3}$ and $w=10 \pi$.

$\varepsilon=10^{-1}$ and $w=5 \sqrt{3}$ are chosen to match the test case conditions For $q=4$ and 6 the direct comparison with the results reported for the SBP methods shows that both methods present similar discretization errors and the same convergence rate. It is also observed that for a given number of nodes $N$, Chebyshev collocation methods present the smallest spatial discretization errors. Figure 11(b) compares the proposed finite-difference methods with the mapped Chebyshev collocation methods proposed by Kosloff and Tal-Ezer The employed values for the mapping parameter $\alpha$ are obtained from resolution considerations $\quad . \alpha_{\mathbf{r}}=\cos (\pi / N)$, accuracy considerations $\quad \alpha_{\mathrm{a}}=\cosh \left(\left|\ln \left(10^{-16}\right)\right| / N\right)^{-1}$, and an alternative accuracy criteria proposed by Hesthaven et al. $\quad \alpha_{\mathrm{h}}=\cos (0.5)$. As Mead and Renaut pointed out the choice $\alpha=\alpha_{\mathrm{r}}$ leads to lost spectral accuracy, as can be observed in Figure 11(b). Choosing $\alpha=\alpha_{\mathrm{a}}$ preserves the spectral accuracy, but the resulting mapped method does not differ much from the Chebyshev collocation method. Finally, the value $\alpha_{h}=\cos (0.5)$ proposed by Hesthaven leads to an intermediate behaviour.

For a prescribed error tolerance $\|\mathbf{E}\|=\varepsilon_{\text {tol }}$, the necessary number of nodes $N$ can be obtained for each degree $q$. Figure 12 shows the number of operations $N_{\mathrm{ops}}=\mathscr{O}(q N)$ and spectral radius $\rho$ (A) of the spatial discretization as a function of $q$ and for different error tolerances. The more grid demanding set of parameter values $\varepsilon=10^{-3}$ and $w=10 \pi$ is used in this figure. The initial steep descent reflects the advantage of high-order finite-difference methods in terms of computational cost, especially when small error tolerances are prescribed. However, the presence of a minimum in the computational cost indicates the existence of an optimum degree $q$, which will be different for each problem. Since it is not worth to go beyond that optimum degree, at least not in terms of computational cost, it would be interesting to be able to know or estimate its value a priori.

Table I shows the number of nodes $N$, number of operations $N_{\text {ops }}$, and spectral radius $\rho(\mathbf{A})$ required by the different numerical methods to obtain the solution of the proposed convectiondiffusion problem with a certain error tolerance. Generally, the mapped Chebyshev collocation methods using $\alpha=\alpha_{\mathrm{a}}$ and $\alpha=\alpha_{\mathrm{h}}$ present smaller spectral radii than the Chebyshev collocation method. Table I shows that the proposed finite-difference methods perform very similar to the mapped Chebyshev collocation methods in terms of computational cost. 
Table I. Number of nodes $N$, number of operations $N_{\mathrm{ops}}$, and spectral radius $\rho$ (A) of the spatial discretization of the convection-diffusion problem proposed $\quad$ for different error tolerances $\varepsilon_{\text {tol }}$, result from using the Chebyshev collocation method, mapped Chebyshev collocation methods with two different values of $\alpha$ and the proposed finite-difference methods with the corresponding optimum degree $q$. Problem parameters are $\varepsilon=10^{-3}$ and $w=10 \pi$.

\begin{tabular}{|c|c|c|c|c|c|c|c|c|c|c|c|c|c|}
\hline \multirow[b]{2}{*}{$\varepsilon_{\text {tol }}$} & \multicolumn{3}{|c|}{ Chebyshev } & \multicolumn{3}{|c|}{ Hesthaven: $\alpha_{h}$} & \multicolumn{3}{|c|}{ Kosloff: $\alpha_{a}$} & \multicolumn{4}{|c|}{ Finite differences } \\
\hline & $N$ & $N_{\text {ops }}$ & $\rho(\mathbf{A})$ & $N$ & $N_{\text {ops }}$ & $\rho(\mathbf{A})$ & $N$ & $N_{\text {ops }}$ & $\rho(\mathbf{A})$ & $q$ & $N$ & $N_{\text {ops }}$ & $\rho(\mathbf{A})$ \\
\hline $10^{-2}$ & 24 & 550 & 110 & 18 & 375 & 44 & 23 & 520 & 96 & 6 & 40 & 240 & 69 \\
\hline $10^{-4}$ & 28 & 673 & 151 & 24 & 550 & 72 & 26 & 611 & 120 & 10 & 45 & 450 & 118 \\
\hline $10^{-6}$ & 32 & 800 & 199 & 32 & 800 & 123 & 28 & 673 & 138 & 14 & 48 & 672 & 169 \\
\hline $10^{-8}$ & 35 & 898 & 308 & 42 & 1132 & 210 & 30 & 736 & 158 & 16 & 55 & 880 & 236 \\
\hline $10^{-10}$ & 38 & 997 & 449 & 54 & 1554 & 644 & 31 & 768 & 165 & 20 & 55 & 1100 & 390 \\
\hline
\end{tabular}

\subsection{Two-dimensional wave equation}

For two-dimensional rectangular grids, the piecewise polynomial interpolation to be used by the finite-difference method can be constructed using a tensor product approach interpolation error is then given by

$$
\begin{aligned}
\varepsilon_{i j}(x, y)= & \frac{\pi_{i}(x)}{(q+1) !} \frac{\partial^{q+1} u}{\partial x^{q+1}}(\xi, y)+\frac{\pi_{j}(y)}{(q+1) !} \frac{\partial^{q+1} u}{\partial y^{q+1}}(x, \eta) \\
& -\frac{\pi_{i}(x)}{(q+1) !} \frac{\pi_{j}(y)}{(q+1) !} \frac{\partial^{2 q+2} u}{\partial x^{q+1} \partial y^{q+1}}\left(\xi^{\prime}, \eta^{\prime}\right)
\end{aligned}
$$

where $\xi, \eta, \xi^{\prime}$, and $\eta^{\prime}$ are some points in the interval $\left[x_{s_{i}}, x_{s_{i}+q}\right] \times\left[y_{s_{j}}, y_{s_{j}+q}\right]$. Again, since nothing can be done to improve the regularity of the function to interpolate, only the magnitudes of the polynomial factors $\pi_{i}(x)$ and $\pi_{j}(y)$ can be minimized by properly selecting the interpolation nodes $x_{i}$ and $y_{j}$, which means that the proposed one-dimensional non-uniform grids can directly be used to construct rectangular grids in multiple dimensions with the same properties.

To demonstrate this result, the two-dimensional wave equation

$$
\frac{\partial^{2} p}{\partial t^{2}}=\frac{\partial^{2} p}{\partial x^{2}}+\frac{\partial^{2} p}{\partial y^{2}}
$$

is solved in the domain $\Omega:[0,1] \times[0,1]$ with the following boundary and initial conditions:

$$
\begin{gathered}
\frac{\partial p}{\partial n}(x, y, t)=0 \quad \forall(x, y) \in \partial \Omega \\
p(x, y, 0)=\mathrm{e}^{-a\left((x-0.5)^{2}+(y-0.5)^{2}\right)}, \quad \frac{\partial p}{\partial t}(x, y, 0)=0 \quad \forall(x, y) \in \Omega
\end{gathered}
$$

whose analytical solution is given by the series

$$
p(x, y, t)=\sum_{m=0}^{\infty} \sum_{n=0}^{\infty} p_{m n} \cos (2 \pi m x) \cos (2 \pi n y) \cos \left(2 \pi \sqrt{m^{2}+n^{2}} t\right)
$$



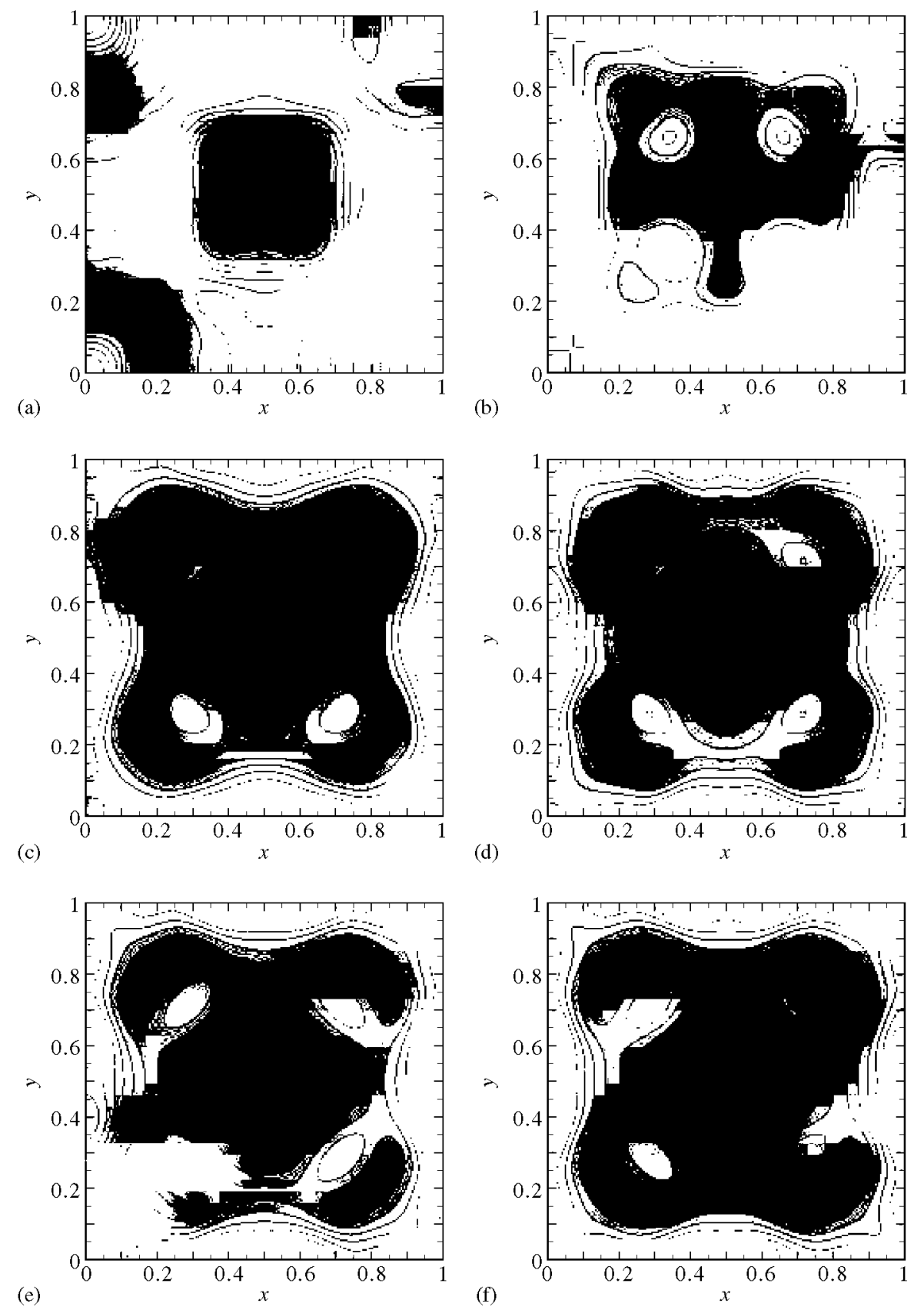

Figure 13. Numerical solutions of the wave equation in a reflecting $2 \mathrm{D}$ box at $t=7.5$ for $a=100$, obtained using the proposed non-uniform grids with $N=20, \Delta t=0.0001$ and (a) $q=2$, (b) $q=4$, (c) $q=6$, (d) $q=8$, and (e) $q=16$. (f) Exact solution. 
with the following expressions, valid when $a \gg 1$, for the coefficients $p_{m n}$ :

$$
p_{m n}=(-1)^{m+n} \frac{d_{m} d_{n} \pi}{a} \mathrm{e}^{-\frac{\pi^{2}}{a}\left(m^{2}+n^{2}\right)}
$$

where $d_{0}=1$ and $d_{i}=2$ otherwise. Using again the same auxiliary variable $v=\partial p / \partial t$, the differential operator in (1) for the variables $(p, v)$ will be

$$
\mathscr{L}(p, v)=\left(v, \frac{\partial^{2} p}{\partial x^{2}}+\frac{\partial^{2} p}{\partial y^{2}}\right)^{\mathrm{T}}
$$

The solution is a two-dimensional wave travelling at constant speed, reflecting at the boundaries, and interfering with itself in the interior of the domain. Figure 13 shows the pressure field $p(x, y, t)$ for $a=100$ at $t=7.5$ computed using the proposed non-uniform grids with different degrees $q$ and $N=20$ : dark grey stands for the lowest value of the pressure and white for the highest value. The computed solutions have been interpolated on finer grids for visualization purposes using the same piecewise polynomial interpolation as in the corresponding computations.

The discontinuities of the interpolant at the subdomain limits are still visible in Figure 13(a), which corresponds to $q=2$. For higher-polynomial degrees, these discontinuities are no longer noticeable, since their amplitudes decay at the same rate as the interpolation error does, as shown in (17). Figure 13(b) shows the solution obtained with $q=4$. Even though there is an improvement with respect to the previous case, the accuracy of the solution is still very poor when compared to the exact solution shown in Figure 13(f). Further increasing the degree of the polynomials improves the accuracy of the solution, as can be seen in Figure 13(c)-(e). Finally, for $q=16$, the structures of the pressure field are well resolved almost everywhere in the computational domain, as shown in Figure 13(e).

Figure 14(a) shows how the spatial discretization errors $|\mathbf{E}|$ are distributed in space at $t=7.5$ for $a=100$ with $N=20$ and $q=10$ : dark grey stands for $|\mathbf{E}|=0$ and white for $|\mathbf{E}|=|\mathbf{E}|_{\max }=0.02$.
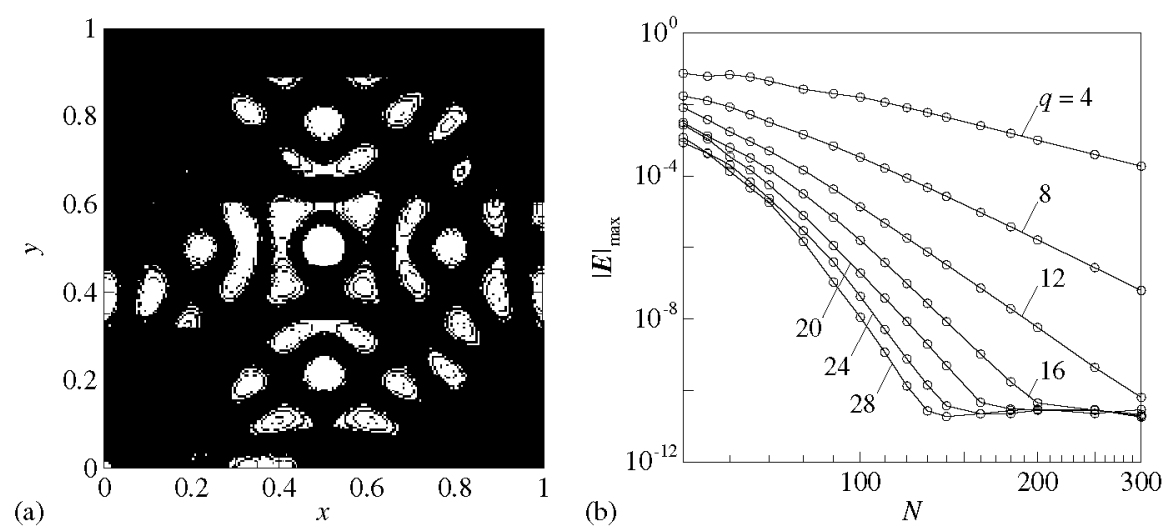

Figure 14. (a) Spatial distribution of discretization errors $|\mathbf{E}|$ at $t=7.5$ for $a=100$ with $N=20$ and $q=10$ : dark grey stands for $|\mathbf{E}|=0$ and light grey for $|\mathbf{E}|=|\mathbf{E}|_{\max }=0.02$. (b) Rate of decay of the maximum discretization error $|\mathbf{E}|_{\max }$ with $N$ for $q=4,8, \ldots, 28$ and $a=500$. 
The spatial structure of the error is associated with the number of nodes of the grid in each direction. Finally, Figure 14(b) shows the rate of decay of the maximum spatial discretization error until round-off with the number of nodes for different values of $q$. The more grid demanding value $a=500$ is used in this plot for visualization purposes.

\section{CONCLUSIONS}

The impact of the node distribution on the pointwise error of piecewise polynomial interpolations has been analysed, and the idea of a non-uniform grid that minimizes that error introduced. An algorithm to obtain the proposed non-uniform grids, given the number of nodes $N$ and the polynomial degree $q$, is presented and used to derive finite-difference approximations to normal and non-normal problems. The following conclusions can be drawn:

1. Finite-difference methods shall use specific grids, exactly as Chebyshev collocation methods do.

2. For $q=N$, the Chebyshev interpolation theory is recovered, rendering Chebyshev collocation methods as a particular case of the proposed finite-difference methods.

3. For $q<N$, the proposed non-uniform nodes are clustered close to the boundaries as a consequence of error control, and not of the problem to be solved.

4. Stable finite-difference approximations to the one-dimensional and two-dimensional wave equations are obtained for all degrees $q$.

5. Finite-difference approximations to the convection-diffusion equation correctly reproduce the transient behaviours of the differential operator for all degrees $q$.

6. The proposed non-uniform grids lead to high-order finite-difference methods which are efficient in terms of computational cost.

Extensions of the proposed finite-difference methods to curvilinear coordinates, using tensor calculus, and to non-linear operators have already been done

while further research is required to better understand the novel aspects of the proposed numerical methods, like the existence of an optimum polynomial degree with respect to the computational cost.

\section{REFERENCES}

Wells VL, Renaut RA. Computing aerodynamically generated noise. Annual Review of Fluid Mechanics 1997; 29:161-199.

Colonius T, Lele S. Computational aeroacoustics: progress on nonlinear problems of sound generation. Progress in Aerospace Sciences 2004; 40(6):345-416.

Moin P, Mahesh K. Direct numerical simulation: a tool for turbulence research. Annual Review of Fluid Mechanics 1998; 30:539-578.

Fletcher CAJ. Computational Techniques for Fluid Dynamics 1: Fundamentals and General Techniques (2nd edn). Springer: Berlin, 1996. 
Canuto C, Hussaini MY, Quarteroni A, Zang TA. Spectral Methods in Fluid Dynamics. Springer: Berlin, 1988. Fornberg B. A Practical Guide to Pseudospectral Methods. Cambridge University Press: Cambridge, U.K., 1998. Boyd JP. Chebyshev and Fourier Spectral Methods (2nd edn). Dover: New York, 2001.

Trefethen LN. Pseudospectra of linear operators. SIAM Review 1997; 39(3):383-406.

Gustafsson B, Kreiss HO, Sundström A. Stability theory of difference approximations for mixed initial boundary value problems II. Mathematics of Computation 1972; 26(119):649-686.

Carpenter MH, Gottlieb D, Abarbanel S. The stability of numerical boundary treatments for compact high-order finite-difference schemes. Journal of Computational Physics 1993; 108:272-295.

Carpenter MH, Gottlieb D, Abarbanel S. Time-stable boundary conditions for finite-difference schemes solving hyperbolic systems: methodology and application to high-order compact schemes. Journal of Computational Physics 1994; 111(2):220-236.

Trefethen LN. Group velocity interpretation of the stability theory of Gustafsson, Kreiss and Sundström. Journal of Computational Physics 1983; 49:199-217.

Strand B. Summation by parts for finite difference approximations for $\mathrm{d} / \mathrm{d} x$. Journal of Computational Physics 1994; 110(1):47-67.

Olsson P. Summation by parts, projections, and stability I. Mathematics of Computation 1995; 64(211):1035-1065. Olsson P. Summation by parts, projections, and stability II. Mathematics of Computation 1995; 64(212): 1473-1493.

Mattsson K, Nordström J. Summation by parts operators for finite difference approximations of second derivatives. Journal of Computational Physics 2004; 199(2):503-540.

Hagstrom T. Experiments with stable, high-order difference approximations to hyperbolic initial-boundary value problems. In Proceedings of the Fifth International Conference on Mathematical and Numerical Aspects of Wave Propagation Phenomena, Bermúdez A, Gómez D, Hazard C, Joly P, Roberts JE (eds). Santiago de Compostela, Spain, 10-14 July 2000.

Zhong X, Tatineni M. High-order non-uniform grid schemes for numerical simulation of hypersonic boundary-layer stability and transition. Joumal of Computational Physics 2003; 190:419-458.

Shukla RK, Zhong X. Derivation of high-order compact finite difference schemes for non-uniform grid using polynomial interpolation. Journal of Computational Physics 2005; 204:404-429.

Hernández JA. High order finite volume methods for the advection-diffusion equation. International Journal for Numerical Methods in Engineering 2002; 53:1211-1234.

Isaacson E, Keller HB. Analysis of Numerical Methods. Dover: New York, 1994.

Ueberhuber CW. Numerical Computation I. Springer: Berlin, 1997.

Trefethen LN. Spectral Methods in MATLAB. SIAM: Philadelphia, PA, 2000.

Kosloff D, Tal-Ezer H. A modified Chebyshev pseudospectral method with an $O\left(N^{-1}\right)$ time step restriction. Journal of Computational Physics 1993; 104(2):457-469.

Mead JL, Renaut RA. Accuracy, resolution, and stability properties of a modified Chebyshev method. SIAM Journal on Scientific Computing 2002; 24(1):143-160.

Hesthaven JS, Dinesen PG, Lynov JP. Spectral collocation time-domain modeling of diffractive optical elements. Journal of Computational Plysics 1999; 155(2):287-306.

Fornberg B. Calculation of weights in finite difference formulas. SIAM Review 1998; 40(3):685-691.

Hildebrand FB. Introduction to Numerical Analysis (2nd edn). Dover: New York, 1987.

Reddy SC, Schmid PJ, Henningson DS. Pseudospectra of the Orr-Sommerfeld operator. SIAM Journal on Applied Mathematics $1993 ; \mathbf{5 3}(1): 15-47$.

Trefethen LN, Embree M. Spectra and Pseudospectra: The Behavior of Nonnormal Matrices and Operators. Princeton University Press: Princeton, 2005.

Greenbaum A. Generalizations of the field of values useful in the study of polynomial functions of a matrix. Linear Algebra and its Applications 2002; 347:233-249.

Reddy SC, Trefethen LN. Pseudospectra of the convection-diffusion operator. SIAM Journal on Applied Mathematics 1994; 54(6):1634-1649.

Berrut JP, Trefethen LN. Barycentric Lagrange interpolation. SIAM Review 2004; 46(3):501-517.

Hermanns M. High order numerical methods applied to the analysis of transport phenomena in combustion. Ph.D. Thesis, Universidad Politécnica de Madrid, 2006. 